\title{
Aspectos epidemiológicos da Leishmaniose Visceral humana e canina em municípios pertencentes à Superintendência Regional de Saúde de Diamantina, Minas Gerais, Brasil (2007- 2012). ${ }^{1}$
}

\section{Epidemiological aspects of human and canine Visceral Leishmaniasis in municipalities of Diamantina Regional Health Superintendence, Minas Gerais State, Brazil (2007-2012).}

\section{Aspectos epidemiológicos de la Leishmaniasis Visceral humana y canina en los municípios pertencentes a la Superintendencia Regional de Salud de Diamantina, Minas Gerais, Brasil (2007-2012).}

\author{
Renata Luiz URSINE ${ }^{2}$ \\ Larissa Ferreira PARANAÍBA ${ }^{3}$ \\ João Victor Leite DIAS ${ }^{4}$ \\ Harriman Aley MORAIS ${ }^{5}$ \\ Herton Helder Rocha PIRES ${ }^{6}$
}

RESUMO: O presente estudo teve como objetivo identificar as áreas de ocorrência e avaliar aspectos epidemiológicos da Leishmaniose Visceral humana e canina nos 33 municípios pertencentes à Superintendência Regional de Saúde de Diamantina no período de 2007 a 2012. Foram coletados dados de notificação dos casos de Leishmaniose Visceral humana, disponibilizados no Sistema de 1 Nota: Este trabalho não teve fonte de financiamento, sendo este resultado da dissertação de mestrado de Renata Luiz Ursine pelo Programa de pós-graduação em Saúde, Sociedade e Ambiente. Conflito de interesse: Os autores declaram não haver nenhum tipo de conflito de interesse.

2 Bióloga. Mestre em Saúde, Sociedade e Ambiente pela Universidade Federal dos Vales do Jequitinhonha e Mucuri. E-mail: renasursine@hotmail.com

3 Bióloga. Mestre em Parasitologia pela Universidade Federal de Minas Gerais. E-mail: larissaparanaiba@bol.com.br 4 Enfermeiro. Doutorando na área de Doenças Infecciosas e Parasitárias pelo programa de pós-graduação em Ciências da Saúde do Centro de Pesquisas René Rachou, FIOCRUZ. E-mail: joaovldias@hotmail.com 5 Médico Veterinário. Doutor em Ciências da Saúde pela Faculdade de Medicina da Universidade Federal de Minas Gerais. Professor da Universidade Federal dos Vales do Jequitinhonha e Mucuri. E-mail: hamorais@gmail.com 6 Biólogo. Doutor em Biologia Parasitária pela Fundação Oswaldo Cruz. Professor Associado II da Universidade Federal dos Vales do Jequitinhonha e Mucuri. E-mail: hhrpires@yahoo.com.br 
Informação de Agravos e de Notificação, e dados de exames sorológicos provenientes de inquéritos caninos registrados em livros da Superintendência Regional de Saúde de Diamantina. No período avaliado foram notificados 79 casos da Leishmaniose Visceral humana, sendo a ocorrência mais comum entre pessoas com idade inferior a 10 anos e igual ou acima de 60 anos, do sexo masculino, residentes em áreas rurais. Araçuaí foi o município que apresentou maior número de notificações da Leishmaniose Visceral humana, sendo classificado como área de transmissão intensa. Quanto à Leishmaniose Visceral canina, 17 municípios enviaram amostras de sangue para realização dos exames e todos apresentaram pelo menos um cão soropositivo. Foi verificado que alguns municípios notificaram a Leishmaniose Visceral em humanos e em cães, outros, notificaram a infecção apenas em cães ou em pessoas e ainda, municípios que não apresentaram nenhuma notificação no período avaliado. Entre os municípios estudados foi possível observar a condução diferenciada da vigilância da Leishmaniose Visceral. Sendo atualmente responsabilidade direta da esfera municipal, cabe ao nível regional orientar e fiscalizar os gestores nas ações de controle de acordo com a situação epidemiológica.

Palavras-chave: Leishmaniose Visceral, Notificação de Doenças, Perfil epidemiológico.

ABSTRACT: This study aimed to identify areas of occurrence and evaluate epidemiological aspects of human and canine visceral leishmaniasis in the 33 municipalities belonging to the Diamantina Regional Health Superintendence, from 2007 to 2012. We collected data regarding human Visceral Leishmaniasis from records available in the Information System and Notification, and canine Visceral Leishmaniasis data from serological surveys recorded in books of Diamantina Regional Health Superintendence. During the study period 79 cases of human visceral leishmaniasis were reported and the most common occurrence was among people under 10 years old and those 60 or more years old, male, residents in rural areas. Araçuaí was the city with the highest number of notifications of human Visceral Leishmaniasis being classified as intense transmission area. For canine leishmaniasis Visceral, 17 municipalities sent blood samples to perform the tests and all had at least one positive dog. It was found that some municipalities have notified visceral leishmaniasis in humans and dogs, others reported infection only in dogs or people and also municipalities that showed no notification during the study period. Among the cities studied was possible to infer the different execution of the surveillance of visceral leishmaniasis. In current health system organization, the control of Visceral Leishmaniasis is a municipal responsibility and the regional management levels should guide and supervise the municipalities according to the epidemiological situation.

Keywords: Leishmaniasis,Visceral, Disease Notification, Health Profile.

RESUMEN: Este estudio tuvo como objetivo identificar las áreas de ocurrencia y evaluar los aspectos epidemiológicos de la leishmaniasis visceral humana y canina en los 33 municipios que pertenecen a la Superintendencia Regional de Salud de Diamantina, entre 2007 y 2012. Se recogieron datos de informes de casos de leishmaniasis visceral humana, disponible en el sistema de información y notificación, y los datos de las encuestas serológicas caninas registrados en los 
libros de la Superintendencia Regional de Salud de Diamantina. Durante el periodo de estudio fueron reportados 79 casos de leishmaniasis visceral humana y la ocurrencia fue más común entre las personas menores de 10 años y las con 60 años de edad o más, del sexo masculino, residentes en las zonas rurales. Araçuaí fue la ciudad con mayor número de notificaciones de leishmaniasis visceral humana y así clasificado como área de transmisión intensa. Sobre la leishmaniasis visceral canina, 17 municipios enviaron muestras de sangre para realizar las pruebas y todos tenían al menos un perro positivo. Se encontró que algunos municipios han notificado la leishmaniasis visceral en humanos y perros, otros informaron la infección sólo en perros o sólo en personas y también hubo los municipios que no mostraron ninguna notificación durante el período de estudio. Entre las ciudades estudiadas fue posible observar diferente seguimiento de la vigilancia de leishmaniasis visceral. Actualmente el control de la leishmanisis visceral está bajo responsabilidad municipal directa, y le corresponde al nivel regional guiar y supervisar a los gerentes de las acciones de control de acuerdo con la situación epidemiológica.

Palabras clave: Leishmaniasis Visceral, Notificación de Enfermedad, Perfil de Salud.

\section{INTRODUÇÃO}

A Leishmaniose Visceral (LV) é causada por protozoários tripanosomatídeos do gênero Leishmania spp., sendo no continente Americano, a principal espécie Leishmania infantum (=L. chagasi), a qual é transmitida por meio da picada de fêmeas de flebotomíneos (Diptera: Psychodidae), sobretudo da espécie L. longipalpis ${ }^{1}$. Devido à sua urbanização, expansão geográfica e alta letalidade em pacientes não tratados, esta doença tem representado um problema de saúde pública mundial ${ }^{2}$.

No continente americano há relatos de ocorrência da LV desde o México até o norte da Argentina ${ }^{3}$, sendo o Brasil responsável por mais de $90 \%$ dos casos registrados neste continente 4 . A incidência anual média da infecção nas Américas no ano de 2012 foi de aproximadamente 4,8 casos para cada 100 mil habitantes, variando de 0,6 casos para cada 100 mil habitantes no México a 5,1 casos a cada 100 mil habitantes no Brasil ${ }^{4}$.

No Brasil, a LV é considerada uma zoonose de difícil controle e ampla distribuição, sendo encontrada nas cinco regiões do país ${ }^{5}$. Originalmente rural, a doença atualmente predomina em aréas urbanas, tendo essa modificação ocorrida principalmente devido aos processos de migração rural-urbana, sobretudo sob condições sociais e econômicas precárias na população ${ }^{6}$. No estado de Minas Gerais entre 2000 e 2010, foram confirmados 5.239 casos humanos, com letalidade de $9,35 \%{ }^{7}$.

Sob o ponto de vista epidemiológico, o cão é considerado como principal reservatório doméstico, sendo, por isso, alvo do programa de controle da LV no Brasil ${ }^{1}$. A importância epidemiológica da LV canina reside no fato de que essa, em geral, precede a LV humana ${ }^{8}$. Nesse sentido, em áreas urbanas 
$182 / /$

há dificuldade em controlar a doença devido ao grande número de cães, muitos dos quais errantes, além das limitações na detecção de cães infectados, visto que boa parte deles é assintomático, não despertando suspeita clínica ${ }^{9}$.

Desta forma, este estudo teve como objetivo identificar as áreas de ocorrência da LV humana e canina nos 33 municípios da Superintendência Regional de Saúde de Diamantina (SRSD), localizados no nordeste do estado de Minas Gerais, abordando aspectos epidemiológicos dessa doença.

\section{METODOLOGIA}

A área selecionada para o estudo compreendeu os 33 municípios sob a jurisdição da SRSD ${ }^{10}$, em sua maioria localizados na região do Vale do Jequitinhonha, Minas Gerais (Figura 1). Esta região tem área de $28.161,308 \mathrm{~km}^{2}$, com uma população estimada de 411.307 habitantes, sendo que desses, 40,55\% residiam em zona rural, de acordo com dados do censo demográfico do Instituto Brasileiro de Geografia e Estatística - IBGE (2010) ${ }^{11}$.

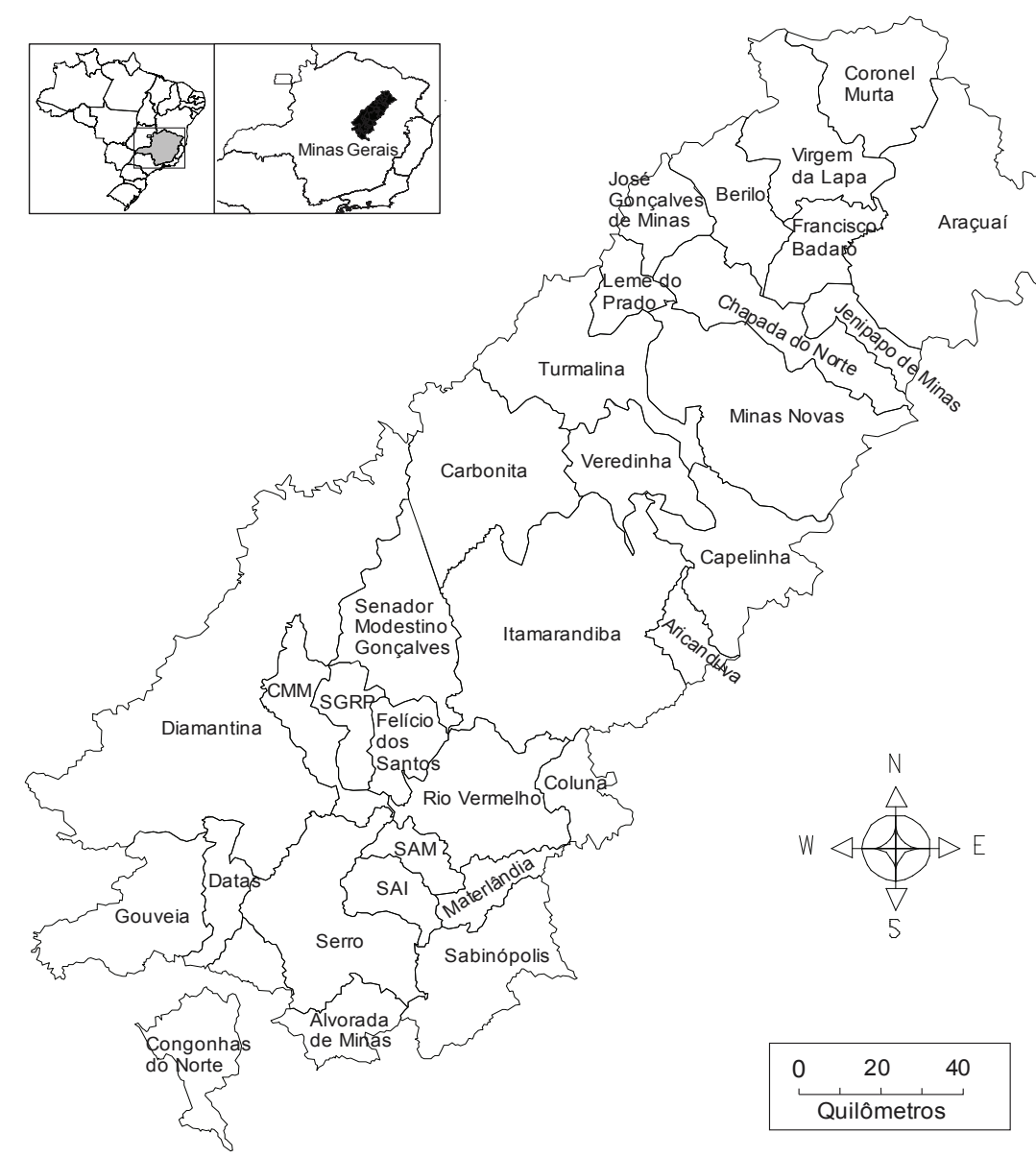

Figura $1-\mathrm{N}$ nantina, no período de 2007 a 2012.

Fonte: elaboração dos autores. 
Nota: CMM: Couto de Magalhães de Minas; SAI: Santo Antônio do Itambé; SAM: Serra Azul de Minas; SGRP: São Gonçalo do Rio Preto.

Os casos de LV humana ocorridos nos municípios da SRSD no período compreendido entre 2007 e 2012 foram coletados na base de dados do Sistema de Informação de Agravos de Notificação (SINAN), em maio de 2014. O recorte inicial no ano de 2007 ocorreu em função de modificações ocorridas no formulário do SINAN a partir deste ano.

Dentre os campos de registro obrigatório no preenchimento da ficha do SINAN, foram coletados os seguintes dados: sexo, idade e zona de residência. Dentre os campos de registros essenciais, porém não obrigatórios, foram coletados dados de evolução do caso. Com base no número de casos apresentados nas fichas de notificação, os municípios foram escalonados quanto ao índice de transmissão da doença de acordo com critérios do Ministério da Saúde ${ }^{12}$, apresentados na tabela 1.

Tabela 1 - Classificação dos municípios quanto à média de casos da Leishmaniose Visceral humana de acordo com critérios do Ministério da Saúde do Brasil (2014).

\begin{tabular}{ll}
\hline Classificação dos municípios & Média de casos de Leishmaniose Visceral humana nos \\
& últimos cinco anos \\
\hline Transmissão intensa & $\geq 4,4$ \\
Transmissão moderada & $\geq 2,4<4,4$ \\
Transmissão esporádica & $<2,4$ \\
Município sem transmissão & Ausência de casos notificados \\
\hline
\end{tabular}

Fonte: Brasil (2014).

Os dados referentes à infecção canina foram coletados em registros de exames sorológicos realizados na rotina do serviço de saúde (inquéritos censitários e/ou amostrais) no período de 2007 a 2012 nos municípios da SRSD. Foram consideradas para esse estudo as seguintes informações: município de origem do cão, localidade, data de realização do exame e resultado do exame.

Os dados foram tabulados no programa Microsoft Excel® 2007, onde também foram calculados os índices percentuais de infecção canina. Os resultados foram analisados de forma descritiva e expressos em tabelas. A distribuição espacial da classificação de transmissão com base nos casos humanos foi representada em mapa temático construído no programa SPRING 5.3 ${ }^{13}$.

Esse trabalho respeitou os aspectos éticos estabelecidos pela resolução 466/12 do Conselho Nacional de Saúde para pesquisas envolvendo seres humanos ${ }^{14}$ tendo sido aprovado pelo Comitê de Ética em Pesquisa da UFVJM, sob número do parecer: 522.035.

\section{RESULTADOS}

Em relação à LV humana foram notificados 79 casos da infecção em 15 municípios. O maior número de casos foi observado entre crianças com idade inferior a 10 anos (25,3\%), seguido de 
pessoas com idade igual ou superior a 60 anos $(22,8 \%)$. Observou-se ainda uma predominância de casos em indivíduos do sexo masculino (62,0\%). Em relação à zona de residência a maioria dos acometidos era residente em área rural $(60,7 \%)$. No que se refere à evolução do caso, destacaramse a alta por cura $(62,0 \%)$ e o não registro da evolução clínica $(24,0 \%)$ (Tabela 2$)$.

Tabela 2 - Perfil dos pacientes com Leishmaniose Visceral humana dos municípios que compõem a Superintendência Regional de Saúde de Diamantina, entre os anos de 2007 a 2012.

\begin{tabular}{|c|c|c|c|c|c|c|c|c|}
\hline Ano & & $\begin{array}{l}2007 \\
(n=6)\end{array}$ & $\begin{array}{c}2008 \\
(n=13)\end{array}$ & $\begin{array}{l}2009 \\
(\mathrm{n}=9)\end{array}$ & $\begin{array}{l}2010 \\
(\mathrm{n}=9)\end{array}$ & $\begin{array}{c}2011 \\
(\mathrm{n}=25)\end{array}$ & $\begin{array}{c}2012 \\
(n=17)\end{array}$ & $\begin{array}{c}\text { Total }(\%) \\
(\mathrm{n}=79)\end{array}$ \\
\hline \multirow{2}{*}{ Sexo } & Masculino & 4 & 8 & 6 & 5 & 16 & 10 & $49(62,0 \%)$ \\
\hline & Feminino & 2 & 5 & 3 & 4 & 9 & 7 & $30(38,0 \%)$ \\
\hline \multirow{5}{*}{$\begin{array}{l}\text { Idade } \\
\text { (anos) }\end{array}$} & $<10$ & 3 & 5 & 4 & 4 & 1 & 3 & $20(25,3 \%)$ \\
\hline & $\geq 10<20$ & 0 & 1 & 3 & 0 & 8 & 2 & $14(17,7 \%)$ \\
\hline & $\geq 20<39$ & 0 & 3 & 0 & 1 & 6 & 5 & $15(19,0 \%)$ \\
\hline & $\geq 39<60$ & 2 & 3 & 1 & 1 & 3 & 2 & 1 \\
\hline & $\geq 60$ & 1 & 1 & 1 & 3 & 7 & 5 & $18(22,8 \%)$ \\
\hline \multirow{5}{*}{$\begin{array}{l}\text { Zona de } \\
\text { residência }\end{array}$} & Urbana & 1 & 7 & 4 & 5 & 9 & 4 & $30(38,0 \%)$ \\
\hline & Rural & 5 & 6 & 5 & 3 & 16 & 13 & $48(60,7 \%)$ \\
\hline & Periurbana & 0 & 0 & 0 & 1 & 0 & 0 & $1(1,3 \%)$ \\
\hline & Cura & 4 & 8 & 5 & 4 & 14 & 14 & $49(62,0 \%)$ \\
\hline & Óbito por LV & 1 & 1 & 0 & 1 & 3 & 0 & $6(7,6 \%)$ \\
\hline \multirow{3}{*}{$\begin{array}{l}\text { Evolução } \\
\text { do caso }\end{array}$} & $\begin{array}{l}\text { Óbito por outra } \\
\text { causa }\end{array}$ & 0 & 0 & 0 & 0 & 1 & 0 & $1(1,3 \%)$ \\
\hline & Transferência & 0 & 1 & 1 & 0 & 1 & 1 & $4(5,1 \%)$ \\
\hline & Ignorado/Branco & 1 & 3 & 3 & 4 & 6 & 2 & $19(24,0 \%)$ \\
\hline
\end{tabular}

Fonte: Sistema de Informação de Agravos de Notificação-SINAN: http://ttr2004.saude.gov.br/sinanweb/tabnet/ dh?sinannet/leishvi/bases/leishvbrnet.def. (Dados coletados em maio de 2014).

Araçuaí apresentou 26 casos de LV entre os anos de 2007 a 2012, sendo o único município classificado como área de transmissão intensa. Berilo foi classificado como área de transmissão moderada. Outros 13 municípios foram classificados como áreas de transmissão esporádica (Figura 2). 


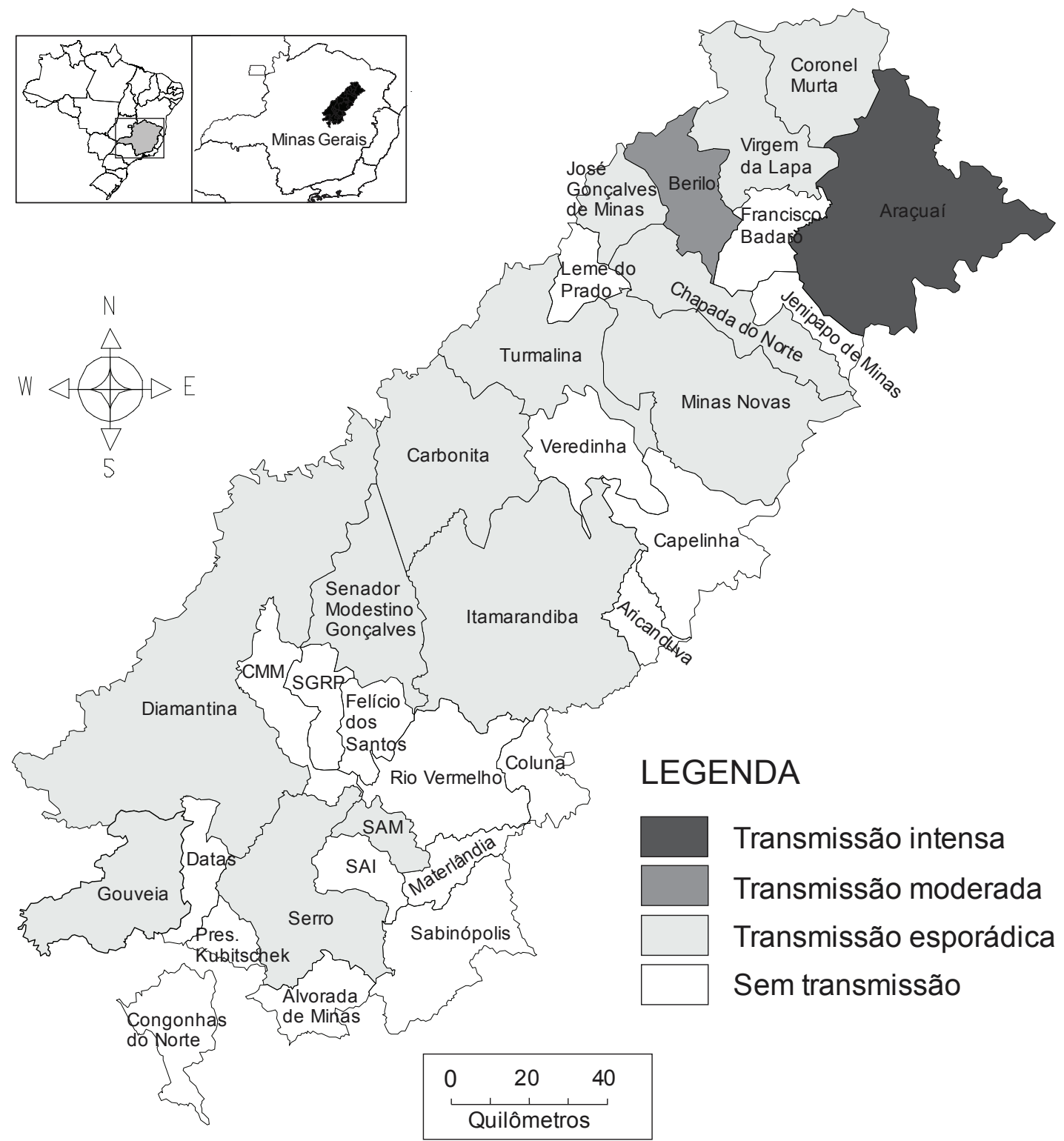

Figura 2 - Municípios pertencentes à SRSD de acordo com a estratificação para transmissão da Leishmaniose Visceral Humana entre 2008 a 2012.

Fonte: Sistema de Informação de Agravos de Notificação - SINAN:

http://dt2004.saude.gov.br/sinanweb/tabnet/dh?sinannet/leishvi/bases/leishvbrnet.def. (Dados coletados em maio de 2014).

Nota: CMM: Couto de Magalhães de Minas; SAI: Santo Antônio do Itambé; SAM: Serra Azul de Minas; SGRP: São Gonçalo do Rio Preto.

Elaboração do mapa: Autores 
Quanto à infecção canina $17(51,5 \%)$ dos 33 municípios enviaram amostras de sangue para realização dos exames, totalizando 6.265 amostras, sendo que destas 451(7,2\%) apresentaram resultados positivos (Tabela 3 ).

Tabela 3 - Municípios que enviaram amostras de sangue à Superintendência Regional de Saúde de Diamantina para a realização de exames de identificação da Leishmaniose Visceral Canina, entre os anos de 2007 a 2012, com seus respectivos valores de positividade da infecção canina.

\begin{tabular}{lccc}
\hline \multicolumn{1}{c}{ Municípios } & Amostras enviadas & $\begin{array}{c}\text { Resultados reagentes } \\
\text { a RIFI* e ELISA** }\end{array}$ & $\begin{array}{c}\text { \% de positividade da } \\
\text { infecção canina }\end{array}$ \\
\hline Alvorada de Minas & 206 & 2 & 1,0 \\
Araçuaí & 635 & 66 & 10,4 \\
Berilo & 135 & 14 & 10,4 \\
Chapada do Norte & 443 & 27 & 6,1 \\
Coronel Murta & 61 & 7 & 11,5 \\
Couto de Magalhães de Minas & 201 & 14 & 7,0 \\
Diamantina & 2235 & 184 & 8,2 \\
Francisco Badaró & 425 & 35 & 8,2 \\
Gouveia & 2 & 1 & 50,0 \\
Itamarandiba & 233 & 5 & 2,2 \\
Jenipapo de Minas & 39 & 3 & 7,7 \\
José Gonçalves de Minas & 32 & 10 & 31,3 \\
Minas Novas & 74 & 6 & 8,1 \\
Presidente Kubitschek & 367 & 24 & 6,5 \\
São Gonçalo do Rio Preto & 135 & 7 & 5,2 \\
Serro & 1022 & 42 & 4,1 \\
Turmalina & 20 & 4 & 20,0 \\
Total & 6.265 & 451 & 7,2 \\
\hline
\end{tabular}

Fonte: Livro de registros de exames sorológicos da Leishmaniose Visceral Canina da Superintendência Regional de Saúde de Diamantina, 2014.

Nota: * RIFI: Reação de Imunofluorescência Indireta. **ELISA: Enzyme Linked Immuno Sorbent Assay (Ensaio imunoenzimático)

Dos 17 municípios com casos positivos para a infecção canina, cinco (Alvorada de Minas, Couto de Magalhães de Minas, Francisco Badaró, Jenipapo de Minas e Presidente Kubitschek) não tiveram notificação da doença humana. Os municípios de Carbonita, Senador Modestino Gonçalves, Serra Azul de Minas e Virgem da Lapa, apresentaram notificação da doença humana, contudo não enviaram material para a realização de diagnóstico da infecção canina.

\section{DISCUSSÃO}

A LV é uma doença endêmica no Brasil acometendo pessoas de todas as idades, sendo que na maior parte das áreas endêmicas a doença ocorre predominantemente entre crianças com idade inferior a 10 anos $^{5,15-19}$. Na SRSD, as crianças menores de dez anos e os idosos (idade superior a 60 anos) representaram os grupos mais acometidos pela LV. Segundo o Ministério da Saúde ${ }^{20}$, as 
crianças nesta idade são mais suscetíveis à infecção por L. infantum devido ao estado de relativa imaturidade imunológica celular, agravado pela desnutrição, muito comum nas áreas endêmicas, além de uma maior exposição ao vetor no peridomicílio. Diferentemente do observado nesse estudo, o acometimento de idosos geralmente é baixo quando comparado com o das crianças, contudo a doença entre estes é preocupante no que se refere à evolução do caso visto que a letalidade nesse grupo é alta ${ }^{1,21}$.

Assim como em outros estudos, a LV na SRSD acometeu principalmente pessoas do sexo masculino ${ }^{15,16,18,19,22-25}$, o que para Prado et al., (2011) ${ }^{5}$ e Brazuna et al., (2012) (22 $^{22}$ pode estar relacionado à maior exposição a ambientes nos quais ocorre transmissão natural da infecção. Já Costa, Pereira e Araújo (1990) ${ }^{26}$ discutem que fatores genéticos e hormonais podem estar relacionados à maior susceptibilidade do sexo masculino à infecção, apesar destas associações ainda não estarem devidamente explicadas.

O fato da maior parte dos casos ter ocorrido em pessoas residentes em áreas rurais $(60,8 \%)$, ao contrário do que vem sendo apresentado em outros estudos ${ }^{6,16,23,27}$, pode ser explicado pelas características demográficas dos municípios da região onde $40,55 \%$ da população residem em áreas rurais.

Quanto à evolução dos casos, o predomínio da alta por cura era esperado, uma vez que os tratamentos atualmente disponíveis oferecem um sucesso na cura da LV em torno de $80 \%{ }^{28-30}$. Além disso, sendo LV uma doença de notificação compulsória, todos os casos diagnosticados devem receber tratamento gratuito pelo Sistema Único de Saúde (SUS).

Por outro lado, o fato do campo referente à evolução dos casos de LV não ser de preenchimento obrigatório $^{31}$ contribuiu para a perda do desfecho em $24,1 \%$ dos casos notificados na SRSD, o que, em última análise, pode mascarar o real número, tanto de curas, como de óbitos. Proporção semelhante de fichas com o desfecho clínico desconhecido foi apresentado pela Organização PanAmericana da Saúde e pela Organização Mundial da Saúde em relação às notificações dos casos de LV no continente americano no ano de 2012, o que evidencia que essa falha não está restrita ao sistema brasileiro ${ }^{4}$. Para que as informações desses sistemas possam ser confiáveis, é importante o treinamento dos técnicos responsáveis pela coleta e abastecimento dos dados ${ }^{32}$ e a realização de análises da qualidade da base dos seus dados regularmente, para que seja possível identificar e solucionar as faltas e as inconsistências nos dados, bem como as duplicidades de registros ${ }^{33}$.

Embora não tenha sido observada sobreposição entre casos humanos e caninos em alguns municípios da região, comparações entre estes devem ser efetuadas de forma cautelosa, uma vez que a amostragem canina realizada nos inquéritos nem sempre representa de forma adequada a população animal ${ }^{34}$. Tal fato também é endossado pela constatação de que 16 municípios sequer realizaram inquérito canino no período estudado, o que pode subestimar um contingente de reservatórios na região. 
Góes, Melo e Jeraldo (2012) ${ }^{15}$ discutem, ainda, o sub-registro de cães soropositivos como reflexo de limitações metodológicas nos programas de controle, uma vez que a busca ativa de cães positivos em algumas áreas, ocorre apenas como resposta à notificação de casos humanos. Outra limitação reside no fato de que muitos cães infectados não são detectados nos exames realizados nos inquéritos, e permanecem como reservatórios ${ }^{35}$.

O Ministério da Saúde ${ }^{12}$ preconiza que caso haja uma pessoa suspeita de estar com LV, devese notificar o caso e realizar a investigação do Local Provável da Fonte de Infecção (LPI), a qual prevê a caracterização da espécie de Leishmania, no caso de nova área de transmissão; realização de levantamento entomológico, no caso de não ter sido verificado a presença do vetor; averiguar se o paciente esteve em locais endêmicos nos últimos seis meses antes do início dos sintomas, bem como a realização da busca ativa dos casos humanos e caninos. Neste sentido, chama atenção o fato de que os municípios de Carbonita, Senador Modestino Gonçalves, Serra Azul de Minas e Virgem da Lapa terem apresentado casos da doença humana, mas não terem apresentado casos da infecção canina e tampouco enviaram material para a realização de exames em cães no período avaliado. Esses dados refletem necessidade de fortalecimento da vigilância e controle da doença nestes municípios, pois a realização de inquéritos caninos é importante para nortear as medidas de vigilância e controle, visto que alguns pesquisadores sugerem que na maioria dos locais a infecção canina precede à humana ${ }^{15,36}$.

As áreas de transmissão intensa e moderada como os municípios de Araçuaí e Berilo, respectivamente, devem realizar $80 \%$ de inquérito canino a cada ano, segundo preconizado pela Secretaria de Estado da Saúde. Para os municípios classificados como áreas de transmissão esporádica, a recomendação é de realizar no mínimo $70 \%$ de inquérito na população canina a cada ano. Já para os municípios silenciosos para a LV humana recomenda-se a realização de no mínimo, $70 \%$ de inquérito canino amostral a cada $\mathrm{ano}^{37}$. Cabe salientar que 12 municípios silenciosos na SRSD não realizaram inquérito canino no período. Garcez et al. (2010) ${ }^{38}$ discutem a importância de se realizar inquéritos temporalmente, sobretudo em áreas silenciosas, pois estas podem ter a presença de cães infectados e por haver pequena densidade de vetores não esteja ocorrendo a transmissão da L. infantum. Nestas condições, eles inclusive recomendam que não seja feita a eutanásia de cães, mas que se mantenha a vigilância entomológica ${ }^{38}$.

Em função do grande número de casos de LV e Leishmaniose Tegumentar Americana, há anos Araçuaí tem sido alvo de pesquisas relacionadas com as leishmanioses ${ }^{39-42}$. Alguns fatores como a criação de animais, presença de vegetação e matéria orgânica no peridomicílio e baixas condições socioeconômicas favorecem a ocorrência da Leishmaniose neste município ${ }^{42}$. No presente estudo, o município de Araçuaí apresentou um alto número de cães infectados e foi o único município classificado como área de transmissão intensa, o que reforça a necessidade de intensificação das ações de controle da LV neste município. 


\section{CONSIDERAÇÕES FINAIS}

A LV representa uma doença endêmica na região da SRSD acometendo principalmente pessoas de áreas rurais, com maior parte dos casos entre crianças e idosos, e do sexo masculino.

O levantamento da ocorrência de LV por meio de fichas do SINAN revelou o recorrente não preenchimento do campo referente à evolução do caso, o que pode levar a subnotificações do desfecho clínico impactando em outros indicadores da doença na região.

Quanto à distribuição da LV humana, Araçuaí foi o único município classificado como área de transmissão intensa e Berilo como de transmissão moderada. Treze municípios enquadraram-se como área de transmissão esporádica e 18 municípios não apresentaram nenhum caso notificado.

Em relação à infecção canina, todos os 17 municípios que realizaram sorologia apresentaram positividade.

Entre os municípios da SRSD é possível observar a condução diferenciada da vigilância da LV. Sendo atualmente tais ações responsabilidade direta da esfera municipal, cabe à SRSD orientar e fiscalizar os gestores de acordo com a situação epidemiológica.

\section{REFERÊNCIAS BIBLIOGRÁFICAS}

1. Marcondes M, Rossi CN. Leishmaniose visceral no Brasil. Braz J Vet Res Anim Sci. 2013; 50(5): 341-352.

2. Moura GS, Santos AM, Aquino DMC, Silva AAM, Caldas AJM. Fatores associados com a infecção assintomática em familiares e vizinhos de pacientes com leishmaniose visceral. Cad Saúde Pública. 2012; 28(12): 2306-2314.

3. Alvar J, Vélez ID, Bern C, Herrero M, Desjeux P, Cano J, et al. Leishmaniasis Worldwide and Global Estimates of Its Incidence. PLoS ONE. 2012; 7(5): e35671. doi:10.1371/journal. pone. 0035671 .

4. Organização Pan-Americana da Saúde; Organização Mundial da Saúde. Leishmanioses. Informe Epidemiológico das Américas. Informe Leishmanioses № 2 - Junho de 2014. Disponível em: http://www.paho.org/hq/index.php?option=com_docman\&task=doc_view\&gid=27090\&Itemid=. Acesso em: 10 de janeiro de 2015.

5. Prado PF, Rocha MF, Souza JF, Caldeira DI, Paz GF, Dias ES. Epidemiological aspects of human and canine visceral leishmaniasis in Montes Claros, State of Minas Gerais, Brazil, between 2007 and 2009. Rev Soc Bras Med Trop. 2011; 44(5): 561-566.

6. Harhay MO, Olliaro PL, Costa DL, Costa CHN. Urban parasitology: visceral leishmaniasis 
in Brazil. Trends Parasitol. 2011; 27(9): 403-409.

7. SES-MG - Secretaria de Estado de Saúde de Minas Gerais. Análise de situação de saúde Minas Gerais 2012. Superintendência de Vigilância Epidemiológica, Ambiental e Saúde do Trabalhador. 2012; pdf, 288p.

8. Ferreira EC, Melo LA, Gontijo CMF. Leishmanioses do Novo Mundo: Estudo de hospedeiros não humanos e sua importância para a compreensão da ecoepidemiologia da doença. Cad Tec Vet Zootec. 2012; 2012(65):09-27.

9. Moshfe A, Mohebali M, Edrissian G, Zarei Z, Akhoundi B, Kazemi B et al. Canine visceral leishmaniasis: Asymptomatic infected dogs as a source of L. infantum infection. Acta Trop. 2009;112(2):101-105.

10. SES-MG - Secretaria de Estado de Saúde de Minas Gerais. SRS Diamantina [homepage na Internet]. Diamantina: SRSD; 28 de Abril de 2011[atualizada em 05 de Janeiro de 2015; [acesso em 06 de jan de 2015]. SRS Diamantina; [aproximadamente 2 telas]. Disponível em: http://www. saude.mg.gov.br/component/gmg/page/213-srs-diamantina-sesmg.

11. IBGE Instituto Brasileiro de Geografia e Estatística. Sinopse do Censo Demográfico 2010 [homepage na Internet]. Brasil: Instituto Brasileiro de Geografia e Estatística [acesso em 06 de jan de 2015]. Disponível em: http://www.censo2010.ibge.gov.br/sinopse/index.php?uf=31.

12. Ministério da Saúde. Manual de Vigilância e Controle da Leishmaniose visceral. Brasília: Ministério da Saúde; 2014.

13. Câmara G, Souza RCM, Freitas UM, Garrido J. SPRING: Integrating Remote Sensing and GIS by Object-Oriented Data Modelling. Computer \& Graphics. 1996; 20(3): 395-403.

14. Ministério da Saúde. Conselho Nacional de Saúde. Resolução no 466, de 2012. Dispõe sobre diretrizes e normas regulamentadoras de pesquisas envolvendo seres humanos. Diário Oficial da União. Brasília: Ministério da Saúde; 2013.

15. Goes MAO, Melo CM, Jeraldo VLS. Série temporal da leishmaniose visceral em Aracaju, estado de Sergipe, Brasil (1999 a 2008): aspectos humanos e caninos. Rev Bras Epidemiol. 2012;15(2): 298-307.

16. BarataRA,PeixotoJC, TanureA, Gomes ME,ApolinárioEC, BodevanEC etal.Epidemiology of Visceral Leishmaniasis in a Reemerging Focus of Intense Transmission in Minas Gerais State, Brazil. Biomed Res Int. 2013; 2013(2013): 405083. http://dx.doi.org/10.1155/2013/405083.

17. Barbosa MN, Carmo RF, Oliveira DCD, Silva GC, Luz ZMP. Atenção aos casos humanos de Leishmaniose Visceral no âmbito da Atenção Primária à Saúde em município da região Tempus, actas de saúde colet, Brasília, 10(1), 179-193, mar, 2016. 
metropolitana de Belo Horizonte. Revista APS. 2013; 16(3): 234-241.

18. Gusmão JD, Brito PA, Leite MTS. Perfil epidemiológico da Leishmaniose Visceral no norte de Minas Gerais, Brasil, no período de 2007 a 2011. Rev Baiana de Saúde Pública. 2014; 38(3): 615-624.

19. Ortiz RC, Anversa L. Epidemiologia da leishmaniose visceral em Bauru, São Paulo, no período de 2004 a 2012: um estudo descritivo. Epidemiol Serv Saúde. 2015; 24(1): 97-104.

20. Ministério da saúde, Secretaria de Vigilância em Saúde. Guia de vigilância epidemiológica. Brasília, DF; 2009.

21. Guimarães IG, Almeida AE. A produção científica brasileira, na última década, sobre a mortalidade de idosos por Calazar. Gestão \& Saúde. 2011; 2(1): 237-248.

22. Brazuna JCM, Silva EA, Brazuna JM, Domingos IH, Chaves N, Honer MR et al. Perfil e distribuição geográfica de casos notificados de leishmaniose visceral na Cidade de Campo Grande, Estado do Mato Grosso do Sul, Brasil, entre 2002 e 2009. Rev Soc Bras Med Trop. 2012; 45 (5): 601-606.

23. Souza VAF, Cortez LRPB, Dias RA, Amaku M, Ferreira Neto JS , Kuroda RBS et al. Análise de aglomerados espaço-temporais da leishmaniose visceral americana em Bauru, São Paulo, Brasil. Cad Saúde Pública. 2012; 28(1): 1949-1964.

24. Batista FMA, Machado FFOA, Silva JMO, Mittmann J, Barja PR, Simioni AR. Leishmaniose: Perfil Epidemiológico dos casos notificados no estado do Piauí entre 2007 e 2011. Revista Univap. [periódico na Internet] 2014 [ acesso em 05 jul 2015] ; 20 (35): 44-55. Disponível em: http:// revista.univap.br/index.php/revistaunivap/article/view/180/196.

25. Cavalcante IJM, Vale MR. Aspectos epidemiológicos da leishmaniose visceral (calazar) no Ceará no período de 2007 a 2011. Rev Bras Epidemiol. 2014; 17(4): 911-924.

26. Costa CHN, Pereira HF, Araújo MV. Epidemia de leishmaniose visceral no estado do Piauí, 1980-1986. Rev Saúde Pública. 1990; 24(5): 361-72.

27. Brazil RP, Pontes MCQ, Passos WL, Fuzari AA, Brazil BG. Lutzomyia (Diptera: Psychodidae:Phebotominae) in the region of Saquarema: potential area of visceral leishmaniasis transmission in the State of Rio de Janeiro, Brazil. Rev Soc Bras Med Trop. 2012; 45(1): 120-121.

28. Barbosa IR. Epidemiologia da Leishmaniose Visceral no estado do Rio Grande do Norte, Brasil. Rev. Epidemiol. Controle Infecç. 2013; 3(1): 17-21.

29. Jardim Filho WR, Cardoso Filho AP, Oliveira Junior JCS, Da Silva MACN, Branco RCC, 
Bezerra GFBet al. Aspectos Clínicos e Laboratoriais da Leishmaniose Visceral no Município de Caxias, MA.News Lab[periódico na Internet]. 2014[acesso em 2014 Ago 12]; 122: 96-104. Disponível em: http://www.newslab.com.br/newslab/revista_digital/122/artigo-6.pdf.

30. Oliveira EN, Pimenta AM. Perfil epidemiológico das pessoas portadoras de Leishmaniose Visceral no município de Paracatu - MG no período de 2007 a 2010. REME. 2014; 18(2): 365-370.

31. Ministério da Saúde, Secretaria de Vigilância em Saúde, Departamento de Vigilância Epidemiológica. Roteiro para uso do sinan net, análise da qualidade da base de dados e cálculo de indicadores epidemiológicos e operacionais leishmaniose tegumentar americana leishmaniose visceral. Brasília, DF; 2008.

32. Rodrigues JRA, Silva-Júnior JLR, Paredes AO, Reis AS, Silva LAC. Doença de Chagas aguda no estado do Maranhão, Brasil: uma comparação entre os bancos de dados do SINAN e da FUNASA. J Manag Prim Health Care. 2013; 4(1): 3-9.

33. Nascimento ES, Maia-Herzog M, Sabroza PC. O acesso público da informação em saúde para as doenças negligenciadas no Brasil. Reciis [periódico na Internet]. 2012 Jun [acesso em Ago 2014 ]; 6(2): [aproximadamente 4 p.]. Diponível em: http://www.reciis.icict.fiocruz.br/index.php/ reciis/article/viewArticle/568/1033.

34. Morais MHF, Fiúza, VOP, Araújo VEM, Carneiro M.Vigilância e controle da leishmaniose visceral no contexto urbano. Cad Tec Vet Zootec. 2012; 2012(65): 44-73.

35. Coura-Vital W. Estudo epidemiológico prospectivo em cães assintomáticos infectados por leishmania (leishmania) infantum e identificação de biomarcadores de infecção. [tese]. Belo Horizonte (MG): Universidade Federal de Minas Gerais; 2011.

36. Silva JP, Werneck GL, Macedo EC, Carvalho H, Cruz MSP. Factors associated with Leishmania chagasi infection in domestic dogs from Teresina, State of Piaú, Brazil. Rev Soc Bras Med Trop. 2012; 45(4): 480-484.

37. SES-MG - Secretaria de Estado de Saúde de Minas Gerais. Instrutivo para Execução e Avaliação das Ações de Vigilância em Saúde: projeto fortalecimento da vigilância em saúde em Minas Gerais (Resolução SES no 4.238/2014). 2014; pdf, 400p.

38. Garcez LM, Cardoso JF, Chagas AP, Miranda JFC, Souza GCR, Soares DC et al. Vigilância da leishmaniose visceral em localidades epidemiologicamente distintas em Juruti, um município minerário do Estado do Pará, Brasil. Rev Pan-Amaz Saúde. 2010; 1(1): 107-116.

39. Gontijo CMF, Silva ES, Fuccio MB, Souza MCA, Pacheco RS, Dias ES et al.Epidemiological studies of an outbreak of cutaneous leishmaniasis in the Rio Jequitinhonha Valley, Minas Gerais, 
Brazil. Acta Trop. 2002; 81(2): 143-150.

40. Dias ES, França-Silva JC, Costa RT, Silva JC, Paula EV, Souza CM et al. Pesquisa de infecção natural por Leishmania em flebotomíneos de área endêmica, município deAraçuaí, MG. In: XIX Reunião Anual de Pesquisa Aplicada em Doença de Chagas - VII Reunião Anual de Pesquisa Aplicada em Leishmanioses: Temas livres Leishmanioses; 2003; Uberaba. Uberaba: Rev Soc Bras Med Trop., 2003.

41. Oliveira FS, Pirmez C, Pires MQ, Brazil RP, Pacheco RS. PCR- based diagnosis for detection of Leishmania in skin and blood of rodents from an endemic area of cutaneous and visceral leishmaniasis in Brazil. Vet Parasitol. 2005; 129 (3-4): 219-227.

42. Santos SEM, Santos HO, Santos RA, Rocha MHF, Silva CP, Sobrinho JFM. Educação ambiental e posse responsável de animais domésticos no combate à Leishmaniose no município de Araçuaí, MG. UDESC em ação [periódico na Internet]. 2013[acesso em 2014 Ago 12]; 7(1): [aproximadamente 3 p.]. Disponível em: http://www.revistas.udesc.br/index.php/udescemacao/ article/view/3289

Artigo apresentado em 08-09-15 Artigo publicado no sistema em 05-02-16 Artigo aprovado em 20-03-16 